\title{
Growth and Characterisation of Lanthanum Doped Sulphamic Acid Single Crystal
}

\author{
B. Kannan ${ }^{1}$, P. R. Seshadri' ${ }^{*}$, P. Murugakoothan ${ }^{2}$ and K. llangovan ${ }^{3}$ \\ ${ }^{1}$ PG and Research Department of Physics, A. M. JAIN College, Meenambakkam, \\ Chennai-600114; seshadri_pr@yahoo.com \\ ${ }^{2}$ MRDL, PG and Research Department of Physics, Pachaiyappa's College, Chennai-600030; \\ murugakoothan03@yahoo.co.in \\ ${ }^{3} \mathrm{PG}$ and Research Department of Physics, RKMVivekananda College, Mylapore, Chennai-600004; \\ ilangokannan@yahoo.com
}

\begin{abstract}
The Lanthanum doped Sulphamic Acid (SA) single crystal was grown by slow evaporation solution growth technique. The Presence of Lanthanum was confirmed by EDAX. The unit cell parameters of the grown crystal were confirmed by single crystal X-ray diffraction. The functional groups of the grown crystal were found by FT-IR study. Thermal and UV-vis absorption studies were performed to know the thermal and optical behaviours of the grown crystal respectively.
\end{abstract}

Keywords: Growth from Solution, X-ray Diffraction, FTIR, Optical Studies, Powder X-ray Diffraction, EDAX

\section{Introduction}

The bulk crystals have a prominent role in the field of science, medicine, engineering, technology and in strategic areas of defense and space science [1]. The search of new organic, inorganic and semi organic non linear materials which have potential applications in photonics, optoelectronics, optical communication, optical image processing is increased in recent times. Suphamic acid and its derivatives have wide industrial applications. The growth, structure determination, neutron diffraction, dielectric, UV-vis-NIR, etching and Raman studies on sulphamic acid single crystal were already reported [2-7]. However no systematic efforts have been made to study the effect of rare earth metals on sulphamic acid. Hence we report here a systematic study on the growth and characterization of Lanthanum doped sulphamic acid (La : SA). The crystalline perfection, structural, presence of funtional groups, thermal, optical behaviours and the incorporation of dopants in (La: SA) are revealed by
X-ray diffraction, EDAX, FTIR, UV-vis-NIR, TG/DTA, studies respectively.

\section{Experimental Procedure}

For the growth of pure SA crystals, the saturated aqueous solution is prepared as per the estimated solubility data, using the recrystallized salt of $\mathrm{SA}$ and the distilled water at $35^{\circ} \mathrm{C}$. A saturated solution of $100 \mathrm{~mL}$ is taken and the solution is filtered. The filtered solution is taken in a beaker, which is tightly closed with perforated filter paper so that the rate of evaporation could be minimized. In order to grow doped crystals, the Lanthanum chloride is added with sulphamic acid in 1:1 molar ratio and dissolved in $100 \mathrm{~mL}$ of double distilled water. The growth run is performed similar to the growth of pure SA crystal. The Figure1(a) and 1(b) show the photographs of pure SA and (La:SA) crystals respectively. The morphology of (La:SA) is changed from that of the pure sulphamic acid single crystal and the visual transparency is also improved after the incorporation of the dopant into the lattice of pure SA crystal.

* Corresponding author:

P. R. Seshadri (seshadri_pr@yahoo.com) 




Figure 1. (a) Pure Sulphamic acid crystals (b) Lanthanum doped Sulphamic acid crystal.

\section{Results and Discussion}

\subsection{EDAX}

The presence of Nitrogen, Oxygen, Sulphur and Lanthanum is confirmed in La: SA single crystal from the EDAX spectrum as shown in Figure 2. The weight percentages of N, $\mathrm{S}, \mathrm{O}$ and $\mathrm{La}$ are given in the Table 1 . From the data it is clear that the amount of lanthanum getting in to the crystal is very low when compared to the amount of lanthanum present in the solution.

\subsection{X-ray Diffraction}

\subsubsection{Single Crystal X-ray Diffraction}

Single crystal X-ray diffraction study is carried out using ENRAF NONIUS FR 590 single crystal X-ray diffractometer. The Sulphamic acid crystal crystallizes

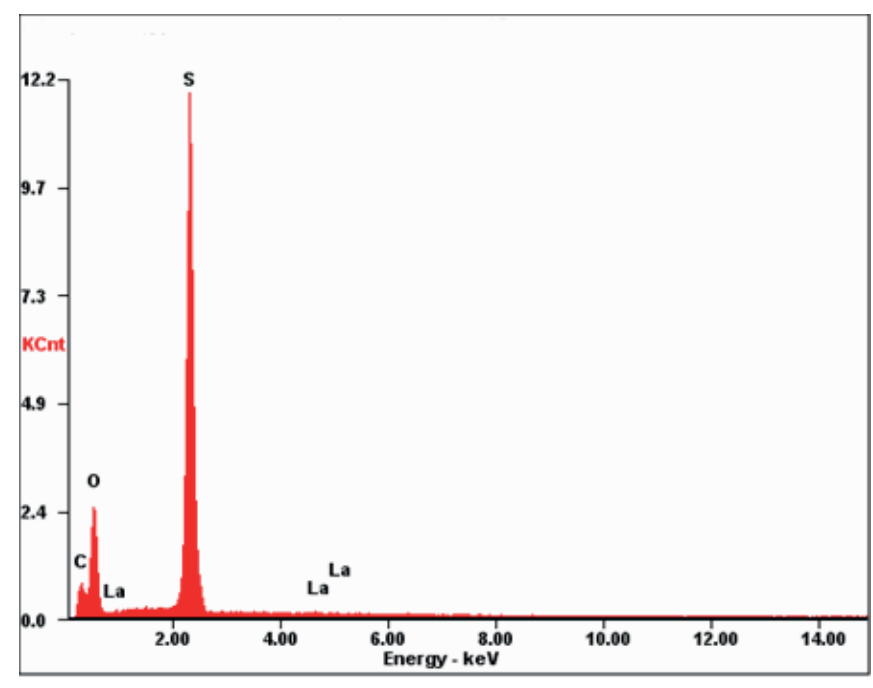

Figure 2. EDAX spectrum of La : SA.

Table 1. Elemental composition of La : SA crystal

\begin{tabular}{ccc}
\hline Element & Wt.\% & At.\% \\
\hline $\mathbf{N}$ & 38.80 & 50.40 \\
$\mathbf{O}$ & 40.87 & 39.86 \\
$\mathbf{S}$ & 19.92 & 09.69 \\
$\mathbf{L a}$ & 00.41 & 00.05 \\
\hline
\end{tabular}

Table 2. Lattice parameters of pure SA and La : SA single crystals

\begin{tabular}{ccc}
\hline Parameters & Pure SA & La : SA \\
\hline $\mathbf{a} \AA$ & 8.100 & 8.050 \\
$\mathbf{b} \AA$ & 8.049 & 8.050 \\
$\mathbf{c} \AA$ & 9.220 & 9.200 \\
$\mathbf{V} \AA^{3}$ & 604.8 & 597 \\
\hline
\end{tabular}

in orthorhombic structure while La : SA single crystal crystallizes in tetragonal structure. The observed lattice parameters are given in table 2.The structural change from orthorhombic to tetragonal by the doping of lanthanum confirms the presence of lanthanum in the crystal lattice of SA crystal.

\subsubsection{Powder $X$ - ray Diffraction}

The powder $\mathrm{X}$ - ray diffraction patterns of pure and doped SA are shown in Figure 3(a) and 3(b). Well defined Bragg peaks are obtained at specific $2 \theta$ angles. The 'd' spacings and ( $\mathrm{h} \mathrm{k}$ l) values for prominent peaks in the spectrum are 
(a)

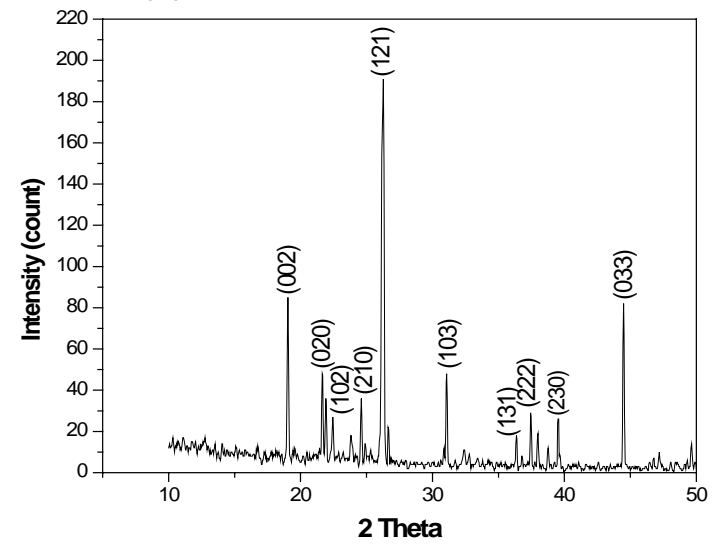

(b)

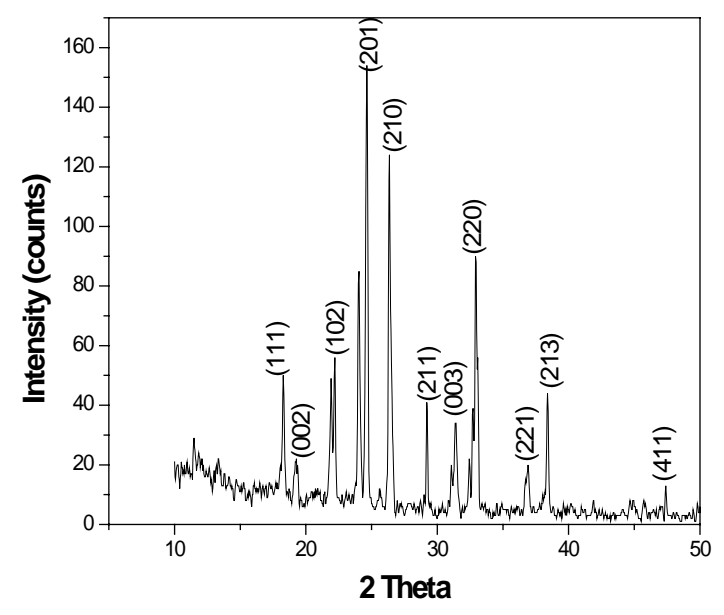

Figure 3. (a) Powder X - ray diffractogram of Pure SA (b) Powder X -ray diffractogram of La : SA.

identified. The extra peaks indexed as (210), (213) and (411) obtained in Figure 3(b) compared with the Figure 3(a) may be due to the incorporation of the lanthanum into the pure SA crystal lattice. Good crystallinity of the grown material is confirmed by the appearance of sharp peaks [8].

\subsection{Fourier Transform Infrared Spectroscopy}

The FTIR spectroscopy study is effectively used to identify the functional groups present in the material. The FTIR spectrum for the La: SA is recorded using BRUKKER IFS $66 \mathrm{v}$ spectrometer by $\mathrm{KBr}$ pellet technique in the range 500 $4000 \mathrm{~cm}^{-1}$ and is shown in Figure 4. The FTIR spectrum of lanthanum doped sulphamic acid seems to be complex because of various functional groups present in the crystal. The functional group assignments for La : SA is summarized in Table 3.

\subsection{UV-vis-NIR Spectroscopy}

The optical absorption spectra of pure SA and La : SA crystals are recorded in the wavelength range 200-800 $\mathrm{nm}$ using Philips PV8700 UV-visible scanning spectrometer. The recorded absorption spectrum is shown in Figure 5. The UV cut off wavelength of pure SA is already reported as $270 \mathrm{~nm}$ [1] whereas (La : SA) crystal has the UV cut off wavelength at $240 \mathrm{~nm}$. Hence it is clear that the presence of lanthanum in SA crystal improves its optical transmission and there is no considerable absorption is found in the range $240 \mathrm{~nm}$ to $800 \mathrm{~nm}$. This clearly indicates that the lanthanum doping has increased the optical window of pure SA crystal.

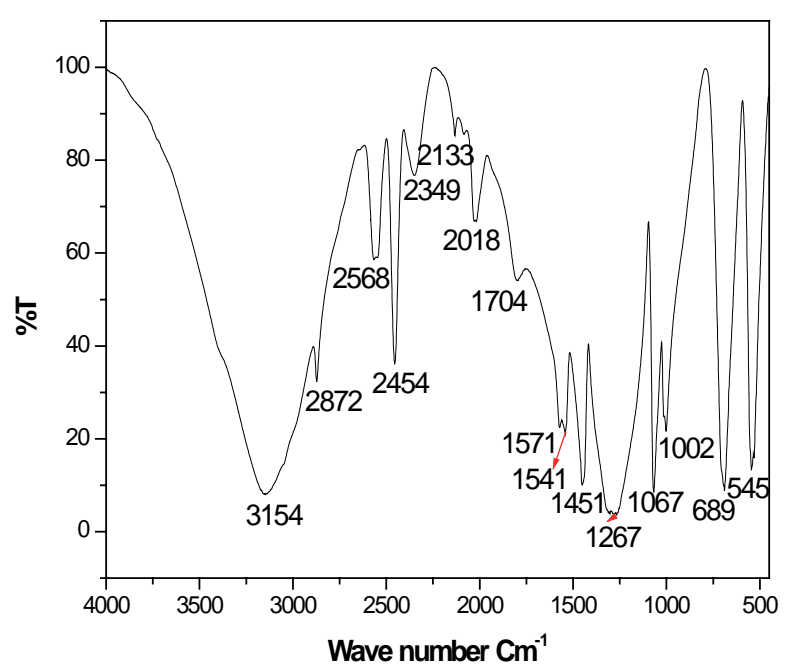

Figure 4. FTIR spectrum of La : SA.

Table 3. Vibrational band assignment for La : SA Single crystal

\begin{tabular}{cl}
\hline Wavenumber $\left(\mathrm{cm}^{-1}\right)$ & Assignment \\
\hline 3154 & Degen. $\mathrm{NH}_{3}{ }^{+}$stretching \\
2872 & Sym. $\mathrm{NH}_{3}^{+}$stretching \\
1541 & Degen. $\mathrm{NH}_{3}{ }^{+}$deformation \\
1451 & Sym. $\mathrm{NH}_{3}^{+}$deformation \\
1267 & Degen. $\mathrm{SO}_{3}^{-}$stretching \\
1067 & Degen. $\mathrm{SO}_{3}^{-}$deformation \\
689 & $\mathrm{NH}_{2}$ and $\mathrm{N}^{-H}$ Wagging \\
545 & Degen. $\mathrm{SO}_{3}$ deformation \\
\hline
\end{tabular}




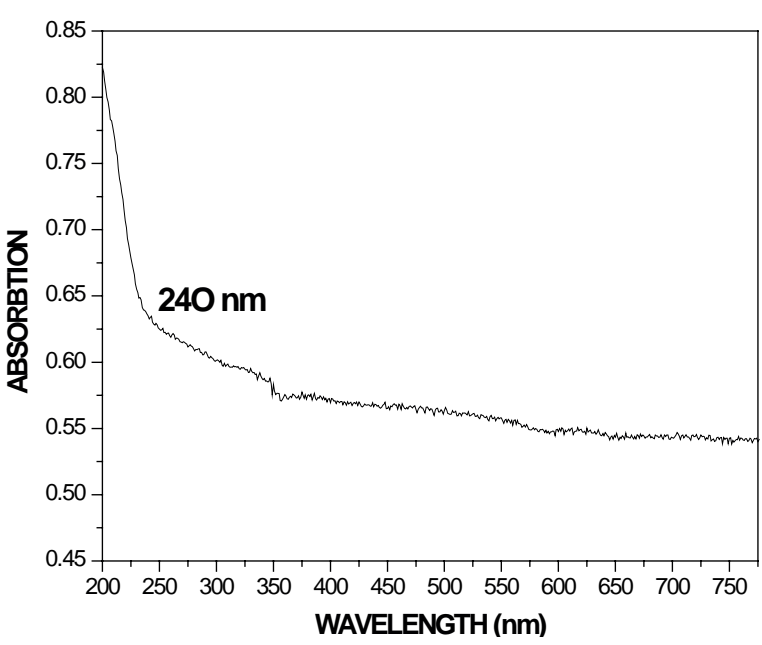

Figure 5. UV-vis-NIR specturm of La : SA single crystal.

\subsection{Thermal studies}

The thermal behaviour of the La: SA crystalline sample is studied using TG and DTA analysis. The thermogravimetric analysis is carried out between $40^{\circ} \mathrm{C}$ and $1400^{\circ} \mathrm{C}$ at a heating rate of $15^{\circ} \mathrm{C} / \mathrm{min}$. in nitrogen atmosphere. The test is carried out using Perkin-Elmer thermal analysis instrument. Alumina is taken as reference material. The TG-DTA thermograms obtained for the dried powder of $\mathrm{La}: \mathrm{SA}$ is shown in Figure 6. From the TG curve it is observed that the La: SA exhibits two stage decomposition. Below $200^{\circ} \mathrm{C}$ there is no detectable weight loss and hence the crystal rejects solvent molecules during crystallization. The first stage of diffused decomposition starts at $225^{\circ} \mathrm{C}$ and ends at $360^{\circ} \mathrm{C}$ in which it undergoes a mass change of about $21.89 \%$ of the initial mass.

The second stage of sharp decomposition takes place at $440^{\circ} \mathrm{C}$ with the mass reduction of $75.68 \%$. These stages of decompositions are well supported by the endothermic peaks at the respective temperatures in the DTA.

\section{6 Nonlinear Optical Test}

A fundamental beam of wavelength $1064 \mathrm{~nm}$ with a pulse duration of $10 \mathrm{~ns}$ and the frequency repetition of $10 \mathrm{~Hz}$ from Q-Switched Nd:YAG laser has been used as a source and passed through the powder sample for second harmonic generation efficiency (SHG) study [9]. Pulse energy and pulse width are maintained as $300 \mathrm{~mJ} \mathrm{~s}^{-1}$ and $10 \mathrm{~ns}$ respectively. The output could be seen as a bright green flash emission from the La: SA but no green emission is

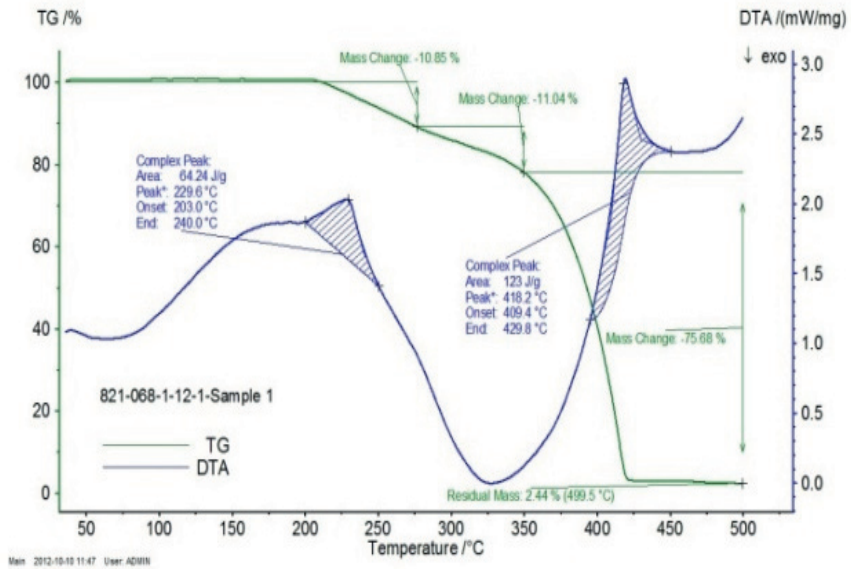

Figure 6. TG - DTA thermograms of La : SA.

observed for pure SA sample. This may be due to the structural change when lanthanum is induced into the pure SA single crystal. From this study it is observed that the dopant induces the NLO property.

\section{Conclusion}

Good optical quality single crystals of pure SA and La : SA have been grown from slow evaporation technique. The crystallinity of the grown sample has been confirmed by $\mathrm{X}$ ray diffraction analysis. Various functional groups present in the grown crystal have been identified by FTIR spectroscopy. The optical transparency has been revealed by UV-vis-NIR study and thermal stability has been confirmed by thermal analysis. Finally it is observed that the dopant induces the NLO property to the grown crystal. Due the broad transmission range and second harmonic generation behavior the La: SA crystal can be used in technologies, such as optical communication, optical computing and dynamic image processing.

\section{References}

1. Babu RR, Ramesh R (2010). Growth, structural, spectral, mechanical and optical properties of pure and metal ions doped sulphamic acid single crystals, Spectrochimica Acta Part A: Molecular and Biomolecular Spectroscopy, vol 76(5), 470 - 475.

2. Rita A, Gharde D et al. (2012), Characterization of sulphamic acid single crystals by optical technique International Journal of Scientific and Research Publications vol 2(6), 2250-3153

3. Sass R L (1960). Acta The neutron diffraction studies on the crystal structure of Sulfamic acid, Cryst., vol 13, part 4, 320-324. 
4. Thaila T, and Kumararaman S (2011). Effect of $\mathrm{NaCl}$ and $\mathrm{KCl}$ doping on the growth of sulphamic acid crystals. Spectrochimica Acta Part A: Molecular and Biomolecular Spectroscopy, vol 82(1), 20-24.

5. Raj A S, and Muthusubramanian P (1982). Normal coordinate analysis of the sulphamate ion, Journal of Molecular Structure: Theochem, vol 89(3-4), 291-296.

6. Hickling S J, and Woolley R G (1990). An ab initio HartreeFock study of the zwitterion of sulphamic acid, +H3NSO3, Chemical Physics Letters, vol 166(1), 43-48.
7. Valluvan, Selvaraju K et al. (2006). Growth and characterization of sulphamic acid single crystals: A nonlinear optical material, Materials Chemistry and Physics, vol 97(1), 81-84.

8. Arumanayagam T, Ananth S et al. (2012). Studies on growth, spectral, optical and mechanical properties of new organic NLO crystal: guanidinium L- glutamate (GuGL). Spectrochimica Acta Part A: Molecular and Biomolecular Spectroscopy, vol 97, 741-745.

9. Vivek P, P.Murugakoothan (2012). Optical and Mechanical Studies on Methyl N- Phenylsuccinamate (MPS). Advanced Materials Research, vol 584, 51-55. 